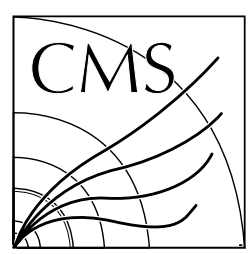

The Compact Muon Solenoid Experiment

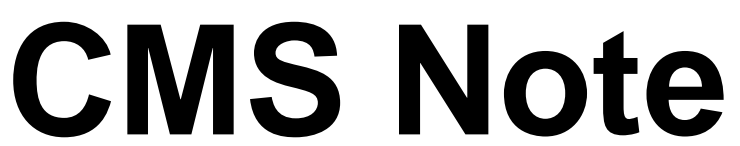

Mailing address: CMS CERN, CH-1211 GENEVA 23, Switzerland

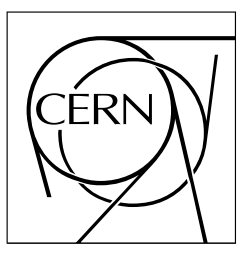

February $23^{\text {rd }}, 2000$

\title{
A Study of the Monitoring of Radiation Damage to CMS ECAL crystals, performed at X5-GIF
}

G. Davies ${ }^{1 \mathrm{a}}$, J.-P. Peigneux ${ }^{2}$, A. Singovski ${ }^{2 \mathrm{a}}$ and C. Seez ${ }^{3}$

\begin{abstract}
The ratio $(\mathrm{R})$, defined as the fractional decrease in the beam signal divided by the fractional decrease in the light monitoring signal, was measured at the GIF-X5 facility for a set of 20 ECAL barrel crystals. The dependence of R on the wavelength of the injected monitoring light and its path-length in the crystals was studied. Measurements of the inter-crystal variation in $\mathrm{R}$ are presented for 10 pre-production crystals. Results on the dependence of signal loss on the irradiation profile are given. The use of lead filters, placed between a ${ }^{137} \mathrm{Cs}$ source and a crystal, to reproduce different longitudinal irradiation profiles has been tested.
\end{abstract}




\section{$1 \quad$ Introduction}

The CMS ECAL is designed to have excellent performance. The target is that the constant term added to the very small intrinsic contribution (from longitudinal leakage) will be limited to $0.5 \%$ [1]. To achieve this the contribution to the constant term from the crystal-to-crystal inter-calibration error must be limited to $0.4 \%$. This will be achieved by E/p calibration using electrons (mainly from W-boson decays) over a period of about one month [1]. However, while the crystals are radiation resistant, there are small residual changes in transparency [2] that occur rapidly for relatively small radiation doses. Recovery (annealing) takes place with a recovery time constant of about one week in the absence of radiation. The resulting changes in signal response must be corrected for, since they occur on a time scale that is short compared to the one month calibration period.

The correction for transparency changes will be made by measuring the crystal transparency, more or less continuously, using a sophisticated monitoring system to inject light pulses into the crystals. Laser light pulses will be fed via quartz fibres into the front of the crystals in the barrel and the rear of the crystals in the endcap. The laser output is monitored close to the crystals being illuminated with PN diodes. The proposed experimental set-up and procedure are described in ref. [1]. But correcting the signal response requires knowledge of the relationship between transmission decrease and shower signal decrease. Previous studies have shown that the decrease in scintillation signal response $\left(\Delta_{\text {particle }}\right)$ is directly proportional to the decrease in the monitoring signal $\left(\Delta_{\text {monitoring }}\right)$. But the ratio $\mathrm{R}=\Delta_{\text {particle }} / \Delta_{\text {monitoring }}$ has been measured in the test beam to have values between 1.5 and 3.0 [3]. It is hoped that production crystals will show little variation in the value of $\mathrm{R}$ - otherwise the value of $\mathrm{R}$ would become another calibration constant, and would have to be measured for each crystal. This would have to be done using in situ calibration data and would be likely to cause some delay in reaching adequate control over the ECAL calibration. (An initial Monte-Carlo study, using test beam data, suggested that it might be achieved in one year of running at low luminosity [4]).

The variation in $\mathrm{R}$ has to be limited to about $5 \%$ to avoid $\mathrm{R}$ becoming another calibration constant needing to be measured for each individual crystal. This can be seen by considering the influence of $R$ on the energy resolution. If $E_{i}$ is the reconstructed energy in a given crystal, $E_{i}=c_{i}(t) \times A_{i}$, where $A_{i}$ is a measured signal and $c_{i}$ is a calibration coefficient. The time variation of $\mathrm{c}_{\mathrm{i}}$ will be corrected using the linear approximation described in the ECAL TDR [1], $\mathrm{c}_{\mathrm{i}}(\mathrm{t})=\mathrm{c}_{\mathrm{i}}\left(\mathrm{t}_{0}\right) \times$ $\left(1+R_{i} \Delta M_{i} / M_{i}\right)$, where $M_{i}$ is the light monitoring signal. The reconstructed energy variation due to $R$ will be $\delta \mathrm{E}_{\mathrm{i}}=\mathrm{c}_{\mathrm{i}}\left(\mathrm{t}_{0}\right) \times \mathrm{A}_{\mathrm{i}} \times\left(\Delta \mathrm{M}_{\mathrm{i}} / \mathrm{M}_{\mathrm{I}}\right) \times \delta \mathrm{R}$. Since $\mathrm{c}_{\mathrm{i}}\left(\mathrm{t}_{0}\right) \times \mathrm{A}_{\mathrm{i}} \approx \mathrm{E}_{\mathrm{I}}$, we obtain $\delta \mathrm{E} / \mathrm{E}=(\Delta \mathrm{M} / \mathrm{M}) \times \delta \mathrm{R}$, or $\delta \mathrm{E} / \mathrm{E}=[\mathrm{R} \times(\Delta \mathrm{M} / \mathrm{M})] \times \delta \mathrm{R} / \mathrm{R}$. So if we set a limit on the contribution to the intercalibration error from this source at $\delta \mathrm{E} / \mathrm{E}=0.2 \%$, and the crystals show a loss of light under irradiation (as seen by the monitoring system) given by $\Delta \mathrm{M} / \mathrm{M}=5 \%$, and, finally, we take the average value of $\mathrm{R}$ to be 1.5 , then $\delta \mathrm{R} / \mathrm{R}=(\delta \mathrm{E} / \mathrm{E}) /[\mathrm{R} \times(\Delta \mathrm{M} / \mathrm{M})]=2.7 \%$.

This figure of $2.7 \%$ is too small for a number of reasons. Most importantly, we have used a figure for the overall magnitude of the change (i.e. $\Delta \mathrm{M} / \mathrm{M}=5 \%$ ). But, in fact, we are interested in something more like the r.m.s. width of the changes. This may be much less than the overall magnitude during stable running conditions, provided that the recovery time of the crystals is long compared to the LHC machine fill time. There are two other assumptions that could also be modified to make the figure somewhat less demanding. Firstly, we have assumed that we wish to limit the contribution to calibration error from $\mathrm{R}$ variation to $0.2 \%$, but perhaps we could tolerate $0.3 \%$. Secondly, we have assumed that the magnitude of crystal damage corresponds to a $5 \%$ change in 
monitoring signals, but maybe the value for final crystals, at least for the barrel region, will be less than that (even at high luminosity).

Bearing these considerations in mind we suggest $5 \%$ as a target limit for the r.m.s. variation of $\mathrm{R}$ in final production crystals.

Verification that the crystal-to-crystal variation of $\mathrm{R}$ is less than about $3 \%$ requires precision measurement. The work at GIF, described in this note has addressed the problem of making such a measurement. The value of $\mathrm{R}$ was measured for 20 Bogoroditsk crystals, taken from different stages of the optimization of crystal growth technology: five $1998 \mathrm{Nb}$ and La doped crystals, five 1999 double doped crystals and 10 pre-production crystals, produced at the second half of 1999 . The crystals where laterally irradiated with a ${ }^{137} \mathrm{Cs}$ source at a dose rate of $\sim 15 \mathrm{rad} /$ hour. The crystal transparency was tracked by a light monitoring system, and the crystal shower response was measured every hour in a $50 \mathrm{GeV}$ electron beam.

A second issue concerns the absolute value of $\mathrm{R}$ - in particular its distance from unity. While the APDs/VPTs and front-end electronics chosen to read-out the ECAL have been made as stable and radiation tolerant as possible some residual changes are expected during LHC operation. These changes are likely to effect both monitoring and shower signals equally. If such changes took place on time scales smaller than the calibration time scale (i.e. one month) and were not independently monitored then they would cause errors to the correction outlined above to the extent that $\mathrm{R}$ was unequal to 1 . For example: suppose $\mathrm{R}=2$ and a $3 \%$ decrease in monitoring light was observed. If the $3 \%$ decrease was due to radiation we would need to apply a $6 \%$ correction to the scintillation signal. But if the $3 \%$ decrease was due to instabilities in the photodetector or electronics chain which effected both monitoring and scintillation signals equally, then we would need to apply a $3 \%$ correction. If the decrease was caused by an unknown mixture of such effects then the needed correction would have an unknown value between 3 and $6 \%$.

It should be noted that significant changes in the electronics gain are not expected to occur on time scales smaller than the calibration time scale (i.e. one month). Also, it is envisaged that monitoring with light of a second wavelength at which there is negligible loss of crystal transparency due to radiation damage will provide a means of deconvolution in such a situation [1]. Nevertheless, the absolute value of $\mathrm{R}$, and its difference from unity, is an important issue, and we have studied the variation of $\mathrm{R}$ due to the wavelength and the path length of the monitoring light.

A third issue concerns the difference in effect of irradiation as a function of the dose profile. Crystal damage studies have been performed using source irradiation from the front, source irradiation from the side, and by using high energy electron beams. Ultimately we are interested in the result of irradiation by particles from LHC interactions, which give rise to a dose profile, as a function of depth, different from any of the profiles arising from the mentioned modes of irradiation. A lead filter can be placed between the source and a crystal undergoing side irradiation to mimic a different profile. Using the technique of geometrical filters it would be possible to simulate the predicted in situ CMS damage profile. This scheme was tested by attempting to mimic the profile of $100 \mathrm{GeV}$ electron beam irradiation.

The layout of the note is as follows: in Section 2 the set-up and experimental procedures are described, and in Section 3 the data analysis procedure is described and illustrated. Section 4 discusses the values obtained for $\mathrm{R}$ and the inter-crystal variation and Section 5 discusses the wavelength and path length dependence. The verification of the lead filters technique is described in Section 6. In Section 7 the results are summarized. 


\section{Experimental set-up and procedure}

The following description will be brief. A fuller description of the system is given in ref. [5] and the operational procedure is discussed in detail in ref. [6].

The basic set-up is illustrated in Fig. 1. The five crystals with APD readout (Hamamatsu, $215 \mathrm{pF}$ ) are sealed in a light tight, temperature stabilized box. The residual temperature variation is $\pm 0.2^{\circ} \mathrm{C}$. A ${ }^{137} \mathrm{Cs}$ gamma source laterally irradiates five crystals at $\sim 15 \mathrm{rad} / \mathrm{hr}$. Periodically the source shutter is closed and the box is moved into the beam line for calibration with $50 \mathrm{GeV}$ electrons. Monitoring light can be injected from both the back and the front of the crystals. Three monitoring light sources are available: a stabilized blue light emitting diode (LED) with peak emission at 450nm, a xenon lamp with monochromator (which can provide light in $10 \mathrm{~nm}$ spectral slices between 300 and $700 \mathrm{~nm}$ ), and a stabilized dye laser running at $425 \mathrm{~nm}$. The final stability of the monitoring system (after using the monitoring reference correction, where appropriate) is $\sim 0.2 \%$ over a 48 hour period (the typical period of an irradiation).

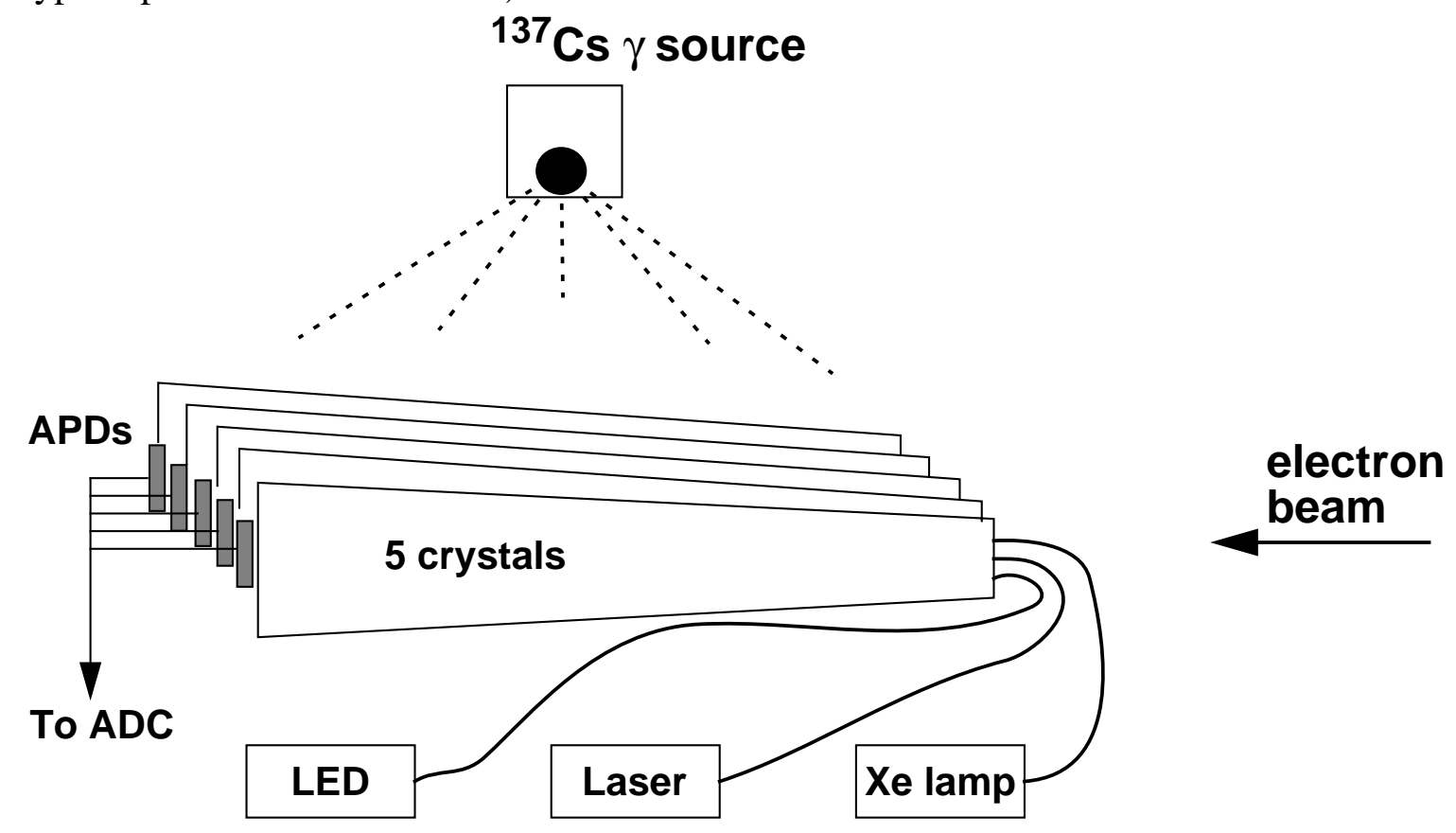

Fig. 1: Sketch of the GIF setup

The measurement sequence for each batch of five crystals is as follows: the crystals are mounted and the system powered up and left to stabilize for about five hours. Each crystal is then calibrated with the electron beam (at a sufficiently low intensity that the resulting dose rate is negligible). This calibration is repeated three times to verify stability and fix the starting point with high precision. The box is then moved into the irradiation position and the source irradiation started. Every hour the source shutter is closed, the box moved to the beam position and the beam signal in each crystal is re-measured. This beam calibration procedure takes about thirty minutes (six minutes per crystal, with about $20 \mathrm{k}$ electrons recorded).

Two one-week periods of test beam were available in 1999, in May and September. In May (Irradiation 18) two batches of crystals were measured. Batch 0 comprised the crystals 2002, 2020, 2022, 2036 and 2045. Batch 1 comprised the crystals 3660, 3662, 3663, 3665 and 3669. Batch 1 was also irradiated using a lead filter designed to reproduce the longitudinal dose profile of $100 \mathrm{GeV}$ 
electron beam irradiation. One crystal in this batch (3662) was also measured while undergoing irradiation using the $100 \mathrm{GeV}$ electron beam run at very high rate (so that the dose rate at shower maximum corresponded to $\sim 15 \mathrm{rad} /$ hour). In September (Irradiation 24) a mixture of the crystals in the two batches was tested to assess our systematic errors (crystals 2022, 2045, 3660, 3662 and 3669). Two batches of very similar final pre-production crystals were also measured - batch 3, made up of crystals 4001, 4002, 4003, 4004, and 4005 and batch 4 made up of crystals 4006, 4007, 4008, 4009 and 4010 .

Batch 0 was also irradiated with the gamma source while light from two similar blue LED light pulse sources was injected from the front and rear of each crystal. This data sequence was used to investigate the dependence of $\mathrm{R}$ on the monitoring light path length.

After each irradiation crystals were annealed, using a standardized procedure, before any subsequent irradiation.

\section{$3 \quad$ Data analysis and results}

The injected light monitoring data provides a continuous measurement of the crystals' optical transmission during ${ }^{137} \mathrm{Cs}$ irradiation. The characteristics of the three light sources are as follows:

- Xenon lamp: slow, about $1 \mu$ s light pulse with narrow, $10 \mathrm{~nm}$ spectral width. Five different wavelengths were used: $375 \mathrm{~nm}, 400 \mathrm{~nm}, 420 \mathrm{~nm}, 460 \mathrm{~nm}$ and $500 \mathrm{~nm}$. The xenon lamp has rather poor amplitude stability (about $\pm 5 \%$ ) and thus a correction, using a reference channel, is required.

- Stabilized blue LED source: relatively slow 500 ns pulse, wide 'gaussian-like' spectrum with the maximum at $450 \mathrm{~nm}$ and a width of about $100 \mathrm{~nm}$, good amplitude stability (better than $0.2 \%)$.

- Laser: fast, $~ 10 \mathrm{~ns}$ pulse at $(425 \pm 1) \mathrm{nm}$, large (10\%) pulse-to-pulse amplitude variation, but good $(0.3 \%)$ mean amplitude stability.

For analysis the monitoring data were split into time slices of five minutes. For each time slice the amplitude of each monitoring signal was histogrammed and a Gaussian distribution fitted to the peak. However, the amplitude distribution of all light monitoring signals is sufficiently Gaussian that the fitted peak position is equal to the simple mean of the complete distribution, and the fitting procedure could be replaced by a simple mean value calculation (see Fig. 2).

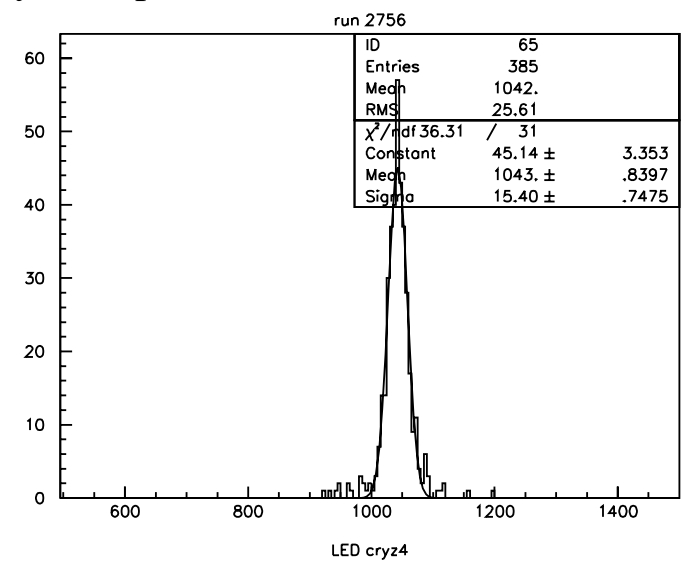

Fig. 2: LED pulse height distribution for a five minute time slice 
The electron beam calibration data were analysed in two steps. The electron position for each event is given by delay wire chambers. First, for each crystal data set, the position of maximum crystal response was found, for each transverse beam coordinate, using a plot of the coordinate from the wire chambers versus the beam signal. Then a window of $10 \times 10 \mathrm{~mm}^{2}$ centred on this point of maximum response ('crystal centre') was selected and the amplitude distribution plotted. The distribution is asymmetric, with a low side tail due to off-centre electrons. A Gaussian was fitted to the peak in the range approximately $-1 \sigma$ to $+2 \sigma$ about the peak position. This procedure is illustrated in Fig. 3 .

The irradiation time (i.e. dose) dependence of the LED and electron signal for one of irradiated crystals is shown in Fig. 4.

run 1976

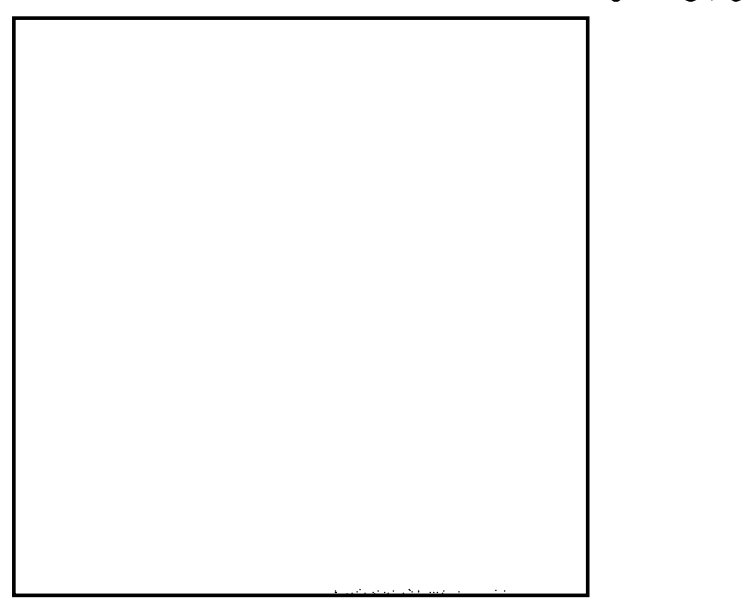

$X$ vs. amp

Y vs. amp

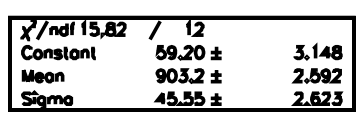

E cryz2

E cryz2 XY

Fig. 3: (above) beam signal versus incident electron position in (left) horizontal and (right) vertical coordinates, (below left) beam signal distribution obtained before and after (shaded histogram) cutting on a window of incident electron position, (below right) shows the distribution after cutting, with an enlarged vertical scale. 


\section{Crystal 3662. Electron / LED ratio}
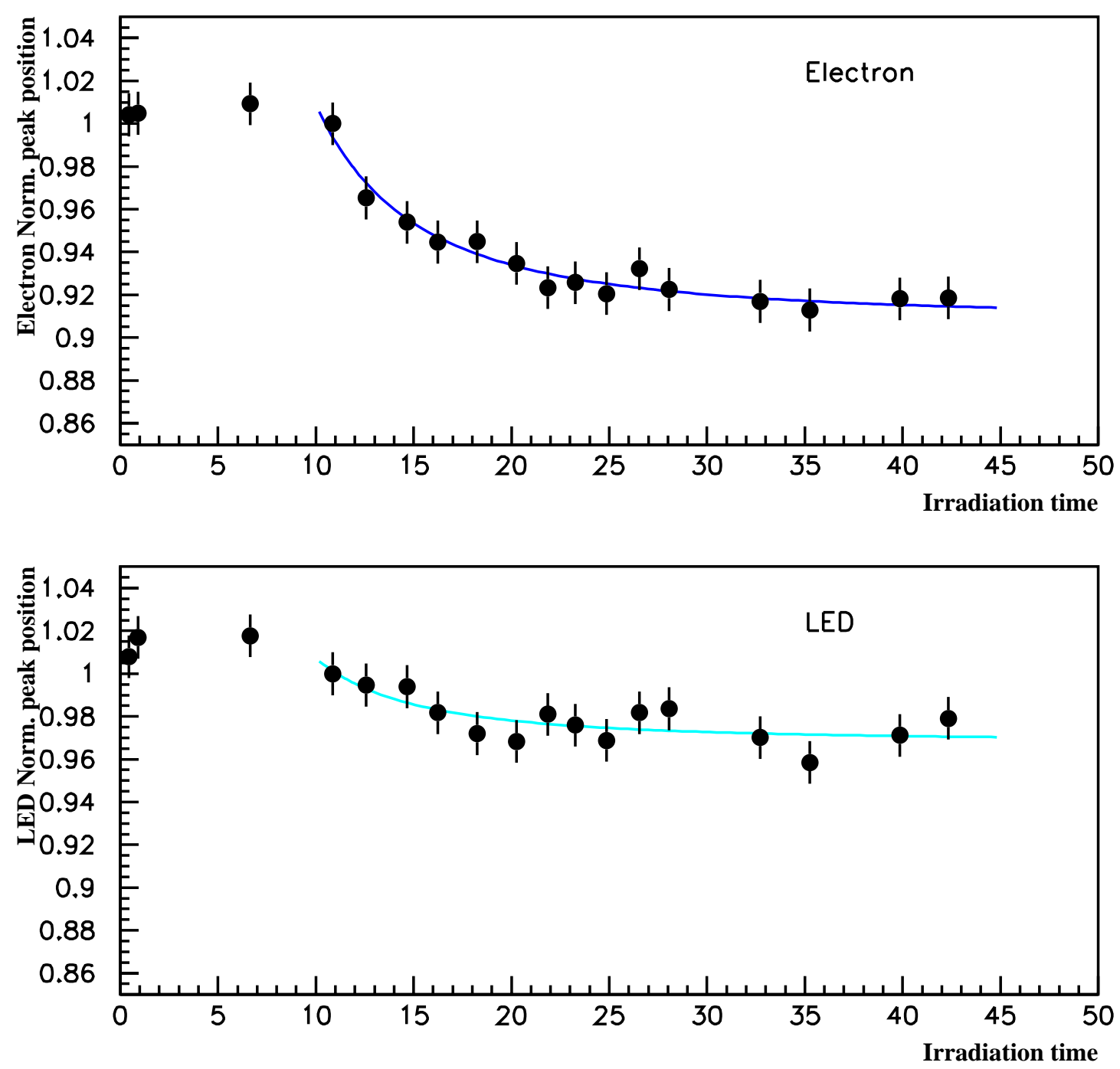

Fig. 4: LED and electron signal as a function of time during irradiation

The changes to the LED and electron signals due to radiation damage which can be seen in Fig. 4 are typical of optimally doped crystals: a small damage of $4-5 \%$ in the first $100-200$ rads and saturation after 300 - 400 rads. The ratio $R$ could be determined, using the plots in Fig. 4, by taking the ratio of electron signal loss divided by the LED signal loss, at saturation. But the precision of this method is limited by the starting point definition. Another way of determining the ratio $\mathrm{R}$ is to fit a straight line to a plot of LED versus electron signal (Fig. 5). This second method was found to give more stable and precise results. 


\section{Irrad 24. $50 \mathrm{GeV}$ electrons. APD readout}

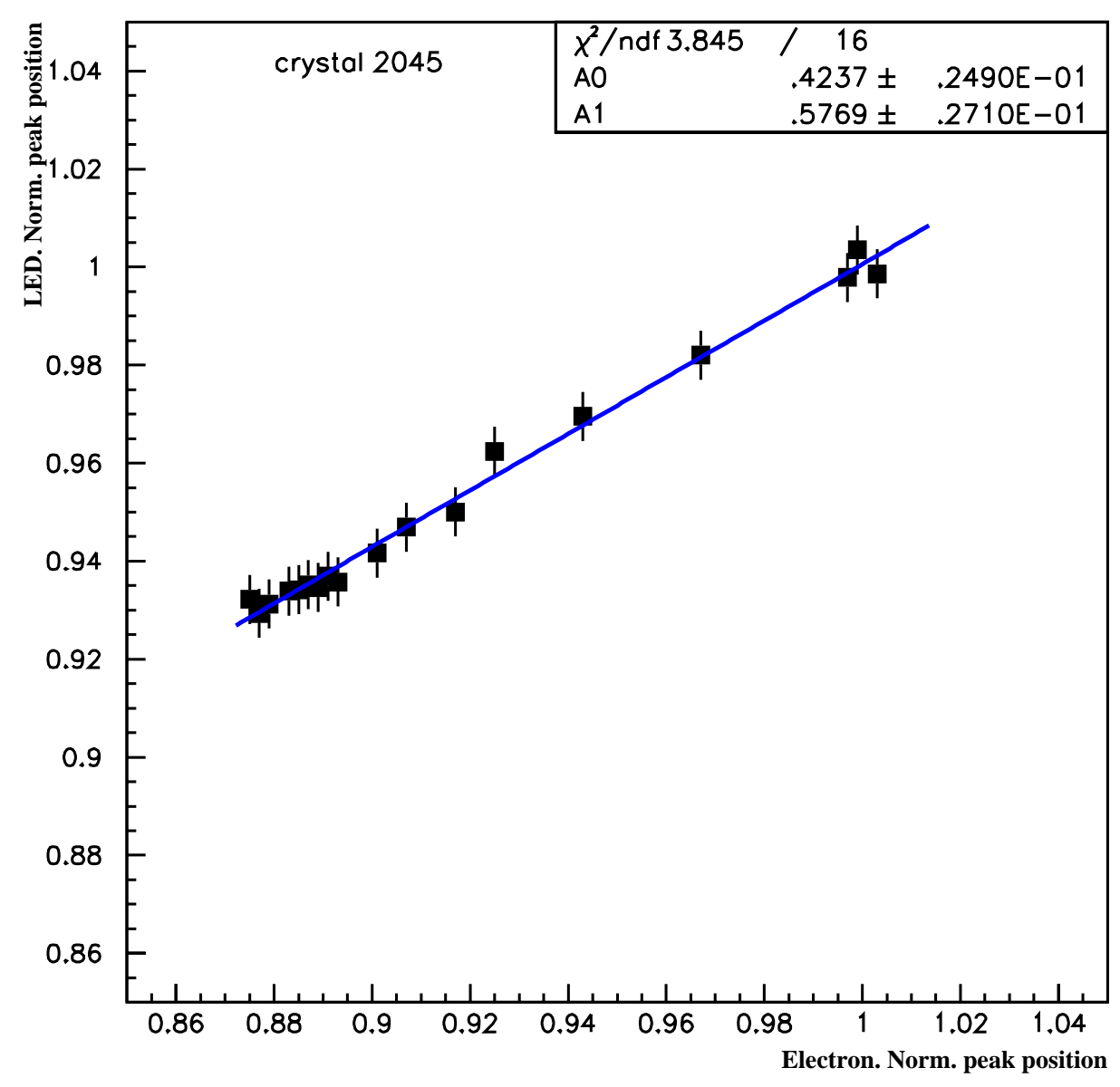

Fig. 5: Normalized LED signal versus normalized electron signal

\section{$4 \quad$ The values obtained for the ratio, $R$}

In Fig. 6 the LED signal is plotted against the electron signal for two different batches of crystals: the R\&D crystals 2002-2045, and the pre-production crystals 4006-4010.

Some improvement of crystal quality, between the two batches, is seen in Fig. 6. The absolute value of the damage has decreased, and there is less crystal to crystal variation of the slopes. The mean value of $\mathrm{R}$ and variation $\left(\Delta \mathrm{R}=\mathrm{R}_{\max }-\mathrm{R}_{\min }\right)$ from the first plot are: $\langle\mathrm{R}\rangle_{2002-2045}=2.1$, $\Delta \mathrm{R}_{2002-2045}=1.4(66 \%)$, from the second one: $\langle\mathrm{R}\rangle_{4006-4010}=1.69, \Delta \mathrm{R}_{4006-4010}=0.24(14 \%)$. The statistical errors on the slope of the straight-line fits are small, 0.03-0.08 for almost all measurements. The dominant error is systematic. It is estimated to be 0.15 and will be discussed in detail below. 
Irrad 18. $50 \mathrm{GeV}$ electrons. APD readout

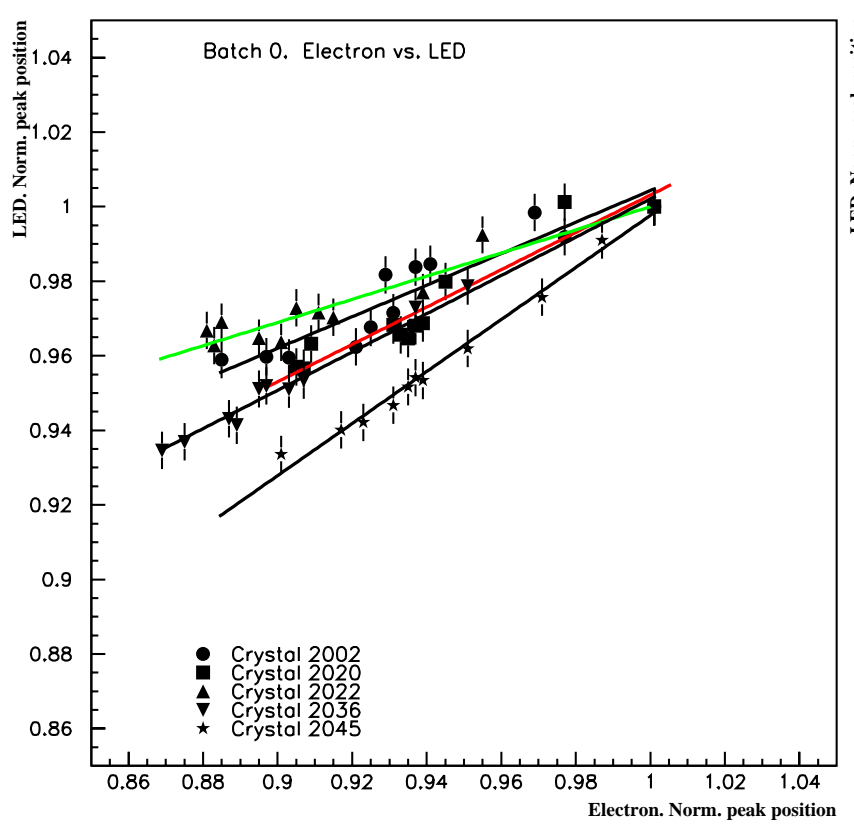

Irrad 24. $50 \mathrm{GeV}$ electrons. APD readout

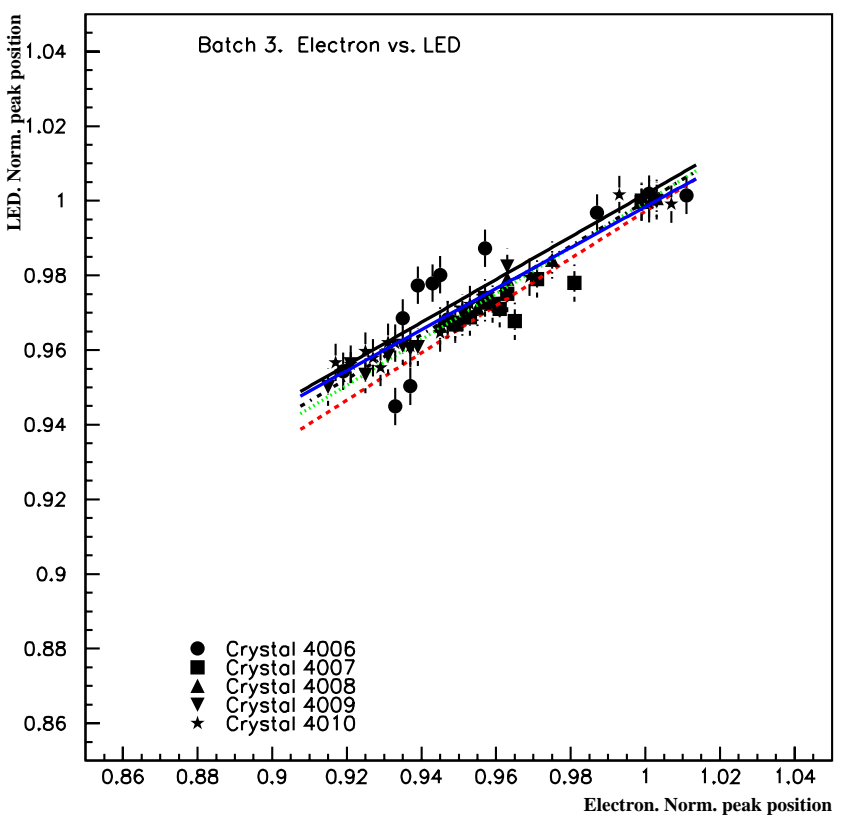

Fig. 6: Normalized LED signal versus normalized beam signal for the crystals 3660-3669 measured in May, and crystals 4006-4010 measured in September

For the ten pre-production crystals $(4001-4010)$ the mean value is: $\langle\mathrm{R}\rangle=1.84 \pm 0.07$ (stat). The r.m.s. width of the R distribution is found to be $\sigma_{R}=0.30 \pm 0.17$, or as a fraction the $\langle R\rangle$ value, $\sigma_{\mathrm{R}} / \mathrm{R}=16 \pm 9 \%$. This is much larger than the desired $3 \%$, but it should be noted that the measurement error is large and dominated by the systematic error. The values of the ratio $\mathrm{R}$ measured in May and September are listed in Table 1 and Table 2.

An attempt was made to correlate the measured value of $\mathrm{R}$ for the pre-production crystals with other crystal properties. No correlation was found with light yield, longitudinal uniformity, longitudinal transmission at $420 \mathrm{~nm}$, slope of longitudinal transmission at turn-on or the variation in UV transversal transmission along the crystal.

Table 1: Electron and LED signal loss for crystals irradiated in May

\begin{tabular}{l|c|c|c|c|c|c|c|c|c|c}
\hline \multicolumn{1}{c|}{ Crystal } & $\mathbf{2 0 0 2}$ & $\mathbf{2 0 2 0}$ & $\mathbf{2 0 2 2}$ & $\mathbf{2 0 3 6}$ & $\mathbf{2 0 4 5}$ & $\mathbf{3 6 6 0}$ & $\mathbf{3 6 6 2}$ & $\mathbf{3 6 6 3}$ & $\mathbf{3 6 6 5}$ & $\mathbf{3 6 6 9}$ \\
\hline \hline e-signal loss (\%) & 11.2 & 9.7 & 10.7 & 12.9 & 9.1 & 5.2 & 8.8 & 7.1 & 5.4 & 4.8 \\
\hline LED signal loss (\%) & 4.8 & 4.6 & 2.9 & 6.8 & 6.4 & 3.1 & 4.4 & 3.6 & 3.2 & 2.3 \\
\hline R (fit to slope) & 2.36 & 2.00 & 3.28 & 1.94 & 1.42 & 1.55 & 2.02 & 1.85 & 1.71 & 1.62 \\
\hline
\end{tabular}


Table 2: Electron and LED signal loss for crystals irradiated in September

\begin{tabular}{l|c|c|c|c|c|c|c|c|c|c}
\hline \multicolumn{1}{c|}{ Crystal } & $\mathbf{4 0 0 1}$ & $\mathbf{4 0 0 2}$ & $\mathbf{4 0 0 3}$ & $\mathbf{4 0 0 4}$ & $\mathbf{4 0 0 5}$ & $\mathbf{4 0 0 6}$ & $\mathbf{4 0 0 7}$ & $\mathbf{4 0 0 8}$ & $\mathbf{4 0 0 9}$ & $\mathbf{4 0 1 0}$ \\
\hline \hline e-signal loss (\%) & 4.6 & 7.6 & 5.1 & 7.3 & 8.2 & 5.7 & 4.1 & 5.2 & 8.1 & 8.0 \\
\hline LED signal loss (\%) & 2.6 & 4.1 & 3.1 & 2.8 & 4.0 & 3.3 & 3.0 & 3.3 & 4.6 & 4.4 \\
\hline R (fit to slope) & 1.78 & 1.88 & 1.57 & 2.60 & 2.10 & 1.75 & 1.58 & 1.63 & 1.68 & 1.82 \\
\hline
\end{tabular}

It is interesting to note that crystals in Table 2 can be grouped, using the value of electron signal loss, into two sets. There are five crystals with the loss greater that $7 \%$ (sample A: crystals 4002, 4004, 4005, 4009 and 4010) and five with a smaller loss (sample B: crystals 4001, 4003, 4006, 4007 and 4008). The mean value and width of $\mathrm{R}$ are respectively:

Sample A: $\langle\mathrm{R}\rangle=2.02 \pm 0.07$ (stat) $\quad 0.15($ sys $), \sigma_{\mathrm{R}}=0.36 \pm 0.17$ (stat+sys), $\sigma_{\mathrm{R}} / \mathrm{R}=18 \pm 9 \%$

Sample B: $\langle\mathrm{R}\rangle=1.66 \pm 0.05$ (stat) $\quad 0.15\left(\right.$ sys), $\sigma_{\mathrm{R}}=0.10 \pm 0.16($ stat + sys $), \sigma_{\mathrm{R}} / \mathrm{R}=6 \pm 10 \%$

The uniformity of the sample B crystals is within the estimated systematic error. The difference between two sets of crystals is believed to be due to details of the doping procedure, and we are told that the sample B procedure will be adopted for the production [7].

\section{Discussion of Errors}

The statistical error on the R measurement comes from the fitting the slope to the LED versus signal loss. This error is $0.03-0.08$ for the majority of measurements, except two cases in May when it was as large as 0.3 due to poor electron peak definition.

The dominant error is systematic. To evaluate this error a set of five crystals $(2022,2045$, 3660, 3662 and 3663) were measured twice, once in May and once in September. The APDs and ADC channels used for these two measurements were different. Two examples, comparing May and September results for two crystals from the five are shown in Fig. 7: crystal 2045 has the best agreement between the two measurements and crystal 3662 the worst. 
Irrad18 vs. Irrad 24.

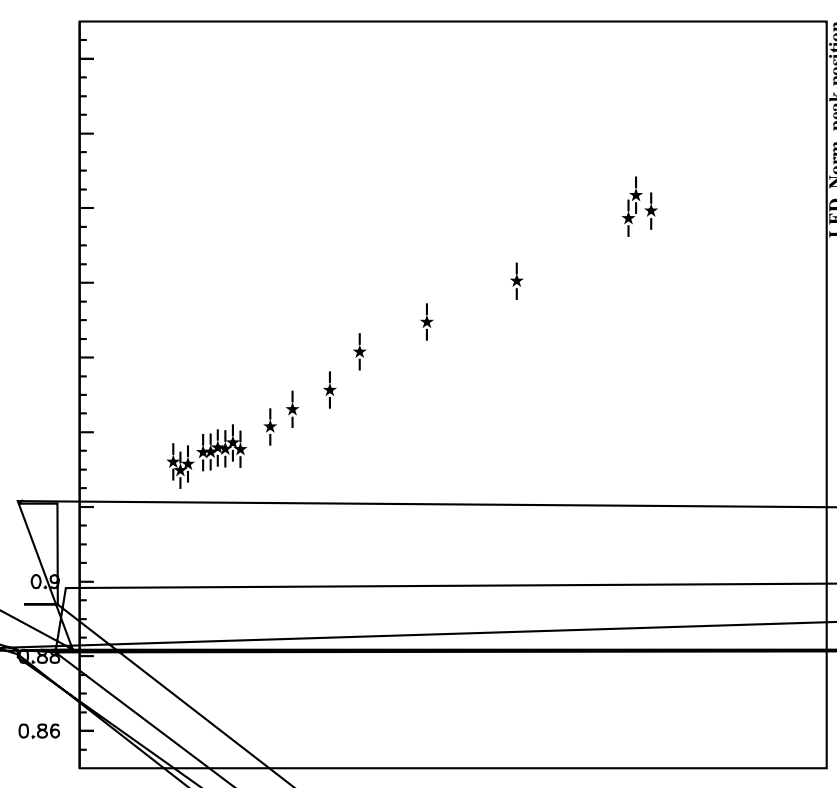

Irrad18 vs. Irrad 24.

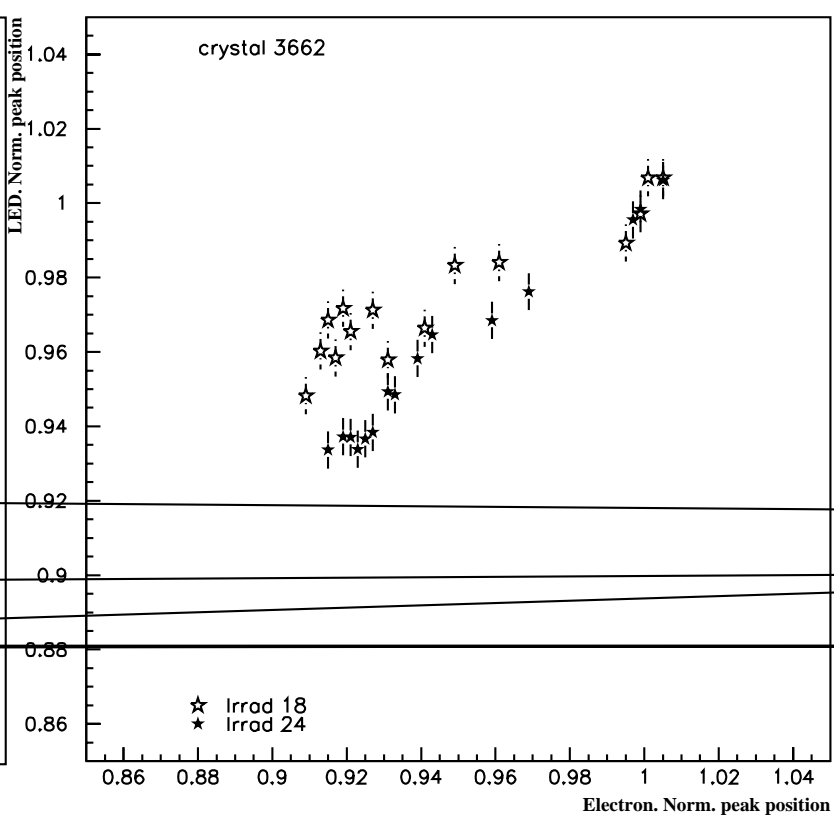

Fig. 7: Normalized electron and LED signals correlation for crystals 2045 and 3662 for May and September runs.

The two measured values of $\mathrm{R}$ for the five crystals are shown in Table 3 . It should be mentioned that the pre-production crystal 3662 has an experimental doping profile and was supposed to be "non-standard".

Table 3: Values of R measured in May and September for the same batch of crystals. The quoted errors are statistical only.

\begin{tabular}{c|c|c|c|c|c}
\hline \multicolumn{1}{c|}{ Crystal } & $\mathbf{2 0 2 2}$ & $\mathbf{2 0 4 5}$ & $\mathbf{3 6 6 0}$ & $\mathbf{3 6 6 2}$ & $\mathbf{3 6 6 3}$ \\
\hline \hline R, May measurement & $3.29 \pm 0.33$ & $1.42 \pm 0.05$ & $1.55 \pm 0.05$ & $2.02 \pm 0.15$ & $1.80 \pm 0.07$ \\
\hline R, September measurement & $2.13 \pm 0.09$ & $1.73 \pm 0.08$ & $1.87 \pm 0.08$ & $1.25 \pm 0.03$ & $1.39 \pm 0.04$ \\
\hline
\end{tabular}

A value for the systematic error cannot be extracted very reliably from this sample of five pairs of measurements, however a rough value can be estimated in the following way: first, a weighted mean of the two measurements is defined for each crystal by:

$$
<\mathrm{R}>=\left(\mathrm{R}_{\text {May }} \mathrm{w}_{\text {May }}+\mathrm{R}_{\text {Sept }} \mathrm{w}_{\text {Sept }}\right) /\left(\mathrm{w}_{\text {May }}+\mathrm{w}_{\text {Sept }}\right), \text { where } \mathrm{w}=1 /\left(\delta_{\mathrm{R}}\right)^{2}
$$

Then the values $\Delta \mathrm{R}_{\mathrm{i}}=\left|\mathrm{R}_{\mathrm{i}}-<\mathrm{R}\right\rangle \mid$ where defined for each measurement. The difference between the maximum and minimum values of $\Delta \mathrm{R}_{\mathrm{i}}$ was then taken to be equal to $4 \sigma_{\text {systematic. This gives an }}$ estimate of the systematic error as about 0.15 , i.e. about $10 \%$ of the mean value of $\mathrm{R}$. However the estimate is dominated by the much less precise May measurement. We believe that the main source of the systematic error is overall system stability, although there is also a possible contribution from procedure used to extract the beam calibration. The $10 \times 10 \mathrm{~mm}^{2}$ window on electron position is 
really too large: if the available statistics allowed a smaller window, we would be able to fit to a more Gaussian signal distribution. The setup stability was greatly improved in September, and some improvements are also foreseen for the beam tests in 2000 . We believe it may be possible to achieve $1-2 \%$ systematic and statistical errors.

\section{$5 \quad$ Wavelength and optical path length dependence}

In section 5.1 we discuss the dependence of $\mathrm{R}$ on the wavelength of the monitoring light, and in section 5.2 its dependence on the monitoring light path length in the crystal. It should be noted that by making these comparisons, of different wavelength and path length, directly during single runs we have removed most sources of systematic error.

\section{Wavelength Dependence}

The values of $\mathrm{R}$ discussed so far were measured using blue LED light pulses injected into the front of the crystal. But data were also taken at other wavelengths using the xenon light source. The values of $\mathrm{R}$ obtained for batch 1 are shown in Table 4. The LED ratio is also given - its emission peak is at $\sim 450 \mathrm{~nm}$ and rather wide.

Table 4: Values of R measured at different wavelengths for crystals in batch 1

\begin{tabular}{l|c|c|c|c|c||c}
\hline \multicolumn{1}{c|}{ Crystal } & $\mathbf{3 6 6 0}$ & $\mathbf{3 6 6 2}$ & $\mathbf{3 6 6 3}$ & $\mathbf{3 6 6 5}$ & $\mathbf{3 6 6 9}$ & $\langle\mathbf{R}\rangle$ \\
\hline \hline LED & 1.50 & 2.12 & 1.85 & 1.71 & 1.62 & 1.89 \\
\hline $\mathrm{Xe} 400 \mathrm{~nm}$ & 0.84 & 1.39 & 0.98 & 0.96 & 1.28 & 1.09 \\
\hline $\mathrm{Xe} 420 \mathrm{~nm}$ & 0.77 & 1.62 & 1.12 & 1.17 & 1.31 & 1.20 \\
\hline $\mathrm{Xe} 460 \mathrm{~nm}$ & 1.09 & 1.95 & 1.46 & 1.26 & 1.57 & 1.47 \\
\hline $\mathrm{Xe} 500 \mathrm{~nm}$ & 1.31 & 3.37 & 1.45 & 2.16 & 2.14 & 2.39 \\
\hline
\end{tabular}

As might be expected the ratio is closer to 1 when the monitoring light is close to the emission peak of the crystal. It has been shown previously that the induced absorption coefficient is larger at shorter wavelengths [8][9].

\section{Path length dependence}

As a consequence of the crystals' high refractive index the monitoring light passing through an air gap from the fibres to the crystal is focused into a cone of half angle $\sim 10^{\circ}$ inside the crystal, whereas the scintillation light is isotropic. It is to be expected that the shorter path length of the monitoring light results in it being less sensitive to loss of transparency than the scintillation signal, resulting in a value of $\mathrm{R}$ larger than unity. To confirm the path length dependence of $\mathrm{R}$ we injected LED light from both front and back of the crystals.

LED light from two identical sources was injected into the front and rear of crystals in batch 0. Fig. 8 shows the decrease in front and back signals for the crystal 2045 as a function of time. 


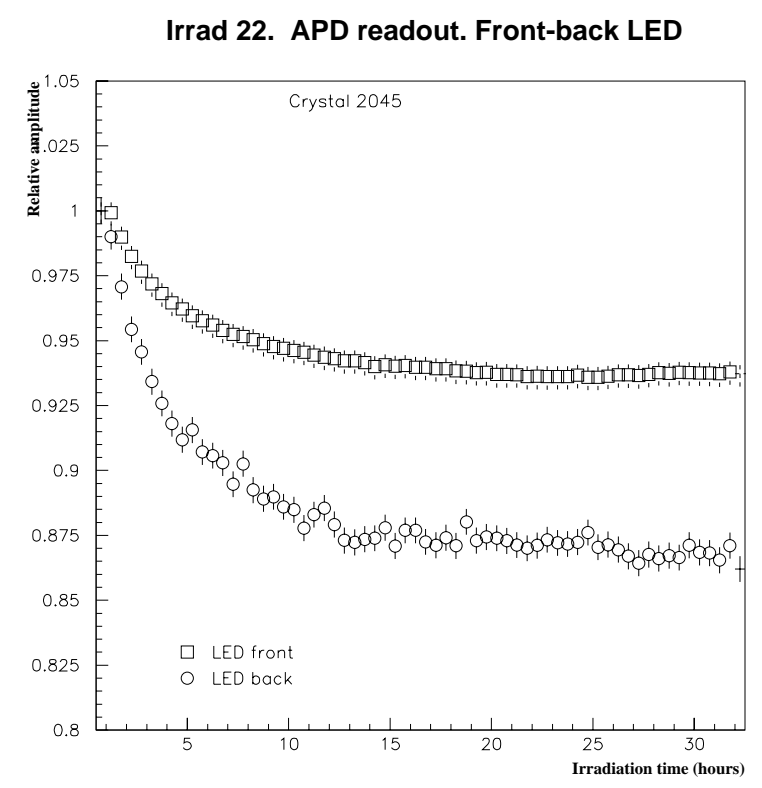

Fig. 8: The development of the LED monitoring signals in crystal 2045 under irradiation. Light pulses from one LED light source are injected in the back of the crystal, and pulses from another are injected from the front.

Table 5 lists, for the five crystals, the ratio of the loss of LED signal seen for light injected from the back of the crystal divided by the loss seen for light injected from the front.

The average ratio between the two values is 2.2 , which is in reasonable agreement with the

Table 5: Ratio of LED signal loss seen for light injected from the back of the crystal divided by the loss seen when the light is injected from the front of the crystal

\begin{tabular}{c|c|c|c|c|c}
\hline Crystal & $\mathbf{2 0 0 2}$ & $\mathbf{2 0 2 0}$ & $\mathbf{2 0 2 2}$ & $\mathbf{2 0 3 6}$ & $\mathbf{2 0 4 5}$ \\
\hline \hline back/front ratio & 2.15 & 2.55 & 2.35 & 1.95 & 2.10 \\
\hline
\end{tabular}

prediction of $2.0 \pm 0.2$ obtained using the ray tracing Monte Carlo program described in ref. [10]. Light injected from the back travels approximately twice the distance through the crystal than light injected at the front does, so the result makes naïve sense.

Since it would be highly desirable to have a value of $\mathrm{R}$ close to one, we are currently investigating the possibility of increasing the path length for light injected at the front by injecting it via a small conical hole in the crystal, which would introduce it into the crystal with a far greater opening angle.

\section{$6 \quad$ Irradiation with Different Dose Profiles}

In order to verify that a specially designed lead filter [11] used with ${ }^{137} \mathrm{Cs}$ source irradiation can mimic the damage caused by a $100 \mathrm{GeV}$ beam, crystal 3662 was irradiated with the ${ }^{137} \mathrm{Cs}$ source from the side with such a lead filter. It was then, after annealing, irradiated with a high intensity electron beam, with the collimators adjusted to give $\sim 15 \mathrm{rad} / \mathrm{hr}$ at the shower maximum. For 
comparison it was also irradiated from the side without lead filters - the standard way that irradiations are performed at GIF, which gives a uniform dose rate of $\sim 15 \mathrm{rad} / \mathrm{hr}$ down the length of the crystal. Fig. 9 shows the electron calibration signal and LED signals as a function of time for the electron beam irradiation, and the uniform ${ }^{137} \mathrm{Cs}$ side irradiation. On each plot the time development of the LED signal values for the filter irradiation are shown for comparison.

One sees that the source with filter reproduces the magnitude of the electron beam damage. The loss resulting from uniform lateral irradiation is about twice as large as that caused by electron beam irradiation. This verification paves the way for use of a filter simulating the LHC irradiation profile to provide a measurement of the signal losses expected during operation at LHC.

Crystal 3662. Cs irradiation

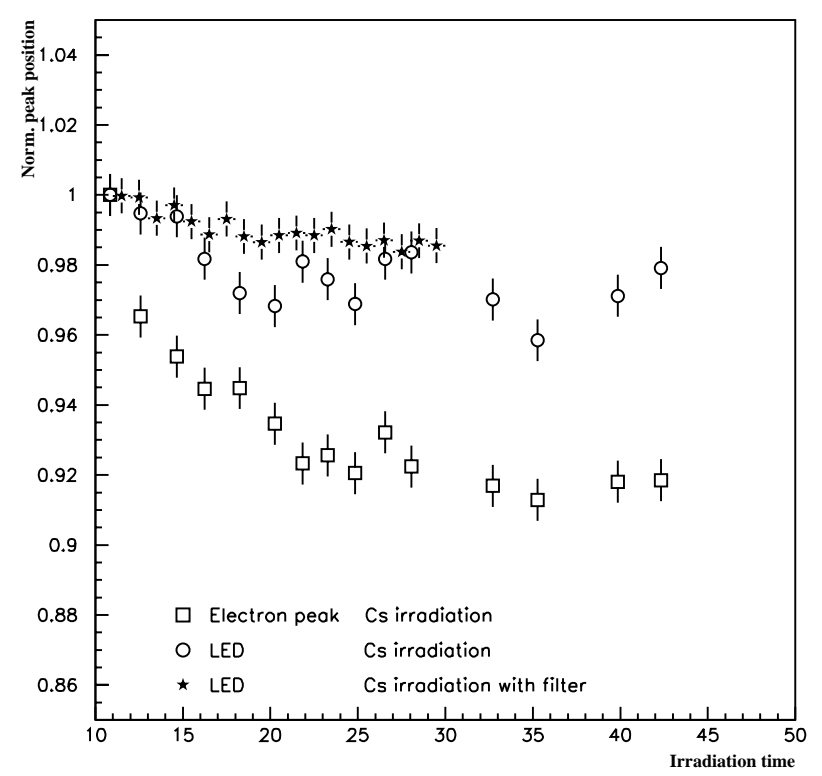

\section{Crystal 3662. Electron $100 \mathrm{GeV}$ irradiation}

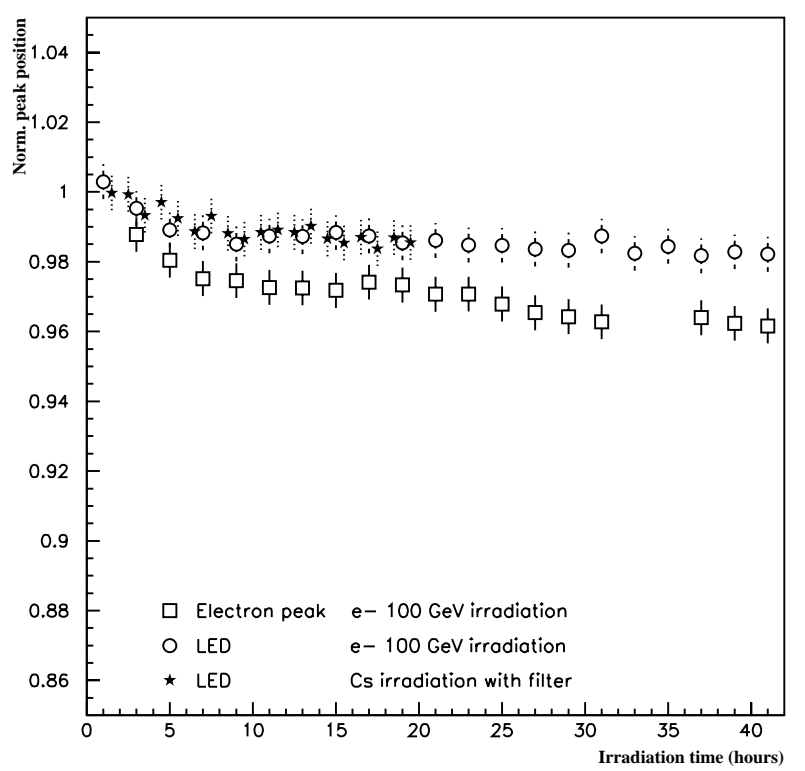

Fig. 9: Signal decrease during two methods of crystal irradiation: (left) electron beam irradiation, (right) uniform ${ }^{137} \mathrm{Cs}$ side irradiation, compared to irradiation with a filter. In both plots the points marked with a black star represent the decrease of the LED signal for a crystal undergoing irradiation through a lead filter designed to mimic the longitudinal dose profile of a $100 \mathrm{GeV}$ electron beam.

\section{$7 \quad$ Summary}

The ratio $(\mathrm{R})$, defined as the fractional decrease in the beam signal divided by the fractional decrease in the light monitoring signal, for crystals undergoing a radiation induced decrease of transparency, has been measured for a number of crystals. Its mean value for a series of preproduction crystals, when using blue LED monitoring light injected into the front of the crystal, is found to be 1.84. It has been confirmed that the value of this ratio depends on both the wavelength of the monitoring light and the path length of this light through the crystal.

For the first time it has been possible to probe the inter-crystal variation in $\mathrm{R}$ for a number of pre-production crystals. The obtained r.m.s. spread is $\sigma_{R} / R=16 \%$ for the crystals $4001-4010$, which is much larger than the $\sim 5 \% \mathrm{R}$ uniformity required if we do not wish to have to measure this value, in situ, for each and every crystal. The spread for the optimally doped crystals sub-set (sample 
B) is $\sigma_{\mathrm{R}} / \mathrm{R}=6 \%$, and is compatible, within the large systematic errors, with the target of $\sim 5 \%$. To clarify this issue this error should be reduced and a larger number of crystals should be tested. An LED has been used here as the monitoring reference, and it will be necessary to check for any possible differences when a monochromatic laser light source of the chosen wavelength of $440 \mathrm{~nm}$ is used.

Dedicated beam time with electrons at GIF-X5 is severely limited. We are currently investigating the possibility of exciting the crystals with a UV laser and obtaining the scintillation response in this way. It would then be possible to be free of the beam time constraints and measure a far larger number of crystals, and be able to study, determine and improve our systematic error with much greater rigour.

\section{$8 \quad$ Acknowledgments}

The authors would like to acknowledge P. Lecoq who insisted on having this installation at the GIF facility for the benefit of the ECAL community, and E. Auffray for her interest and her timely provision of crystals for measurement. Their thanks go also to M. Korzkik for deep and fruitful discussions. We would also like to thank R. Chipaux, Y. Hui, R. Marcos and P. Sempere for their valuable help during part of the data taking. We would like to thank A. Vorvolakos for assistance with part of the data analysis. The authors from LAPP wish to acknowledge A.Feodorov ,A. Lopatik and O.Missevich from INP in Minsk for providing and tuning the Xe lamp and LED light sources used in this work. They also thank A. Dolgopolov, A. Iniakine, P. Shagin and V. Sougonaiev for their help during the building phase of the set up while on a visit to LAPP under the International Scientific Programme of Cooperation (PICS 576 and PICS 762).

\section{$9 \quad$ References}

[1] The Electromagnetic Calorimeter Project, Technical Design Report, CERN/LHCC 97-33 (December 1997).

[2] A. N. Annenkov et al., Systematic Study of the PbW04 Crystal Short Term Instability Under Irradiation, CMS Note 1997/055

[3] E. Auffray et al., Beam Tests of Lead Tungstate Crystal Matrices and a Silicon Strip Preshower Detector for the CMS Electro-magnetic Calorimeter, Nucl. Inst. and Meth., A412 (1998) 223.

[4] C. Seez, private communication.

[5] J.P. Peigneux et al., The X5-GIF Facility, CMS Note 1999/061.

[6] J.P. Peigneux et al., Operational Procedure for the X5-GIF Facility, CMS Internal Note in Preparation,

[7] M. Khorzik, private communication.

[8] E. Auffray et al., Improvement of Several Properties of Lead Tungstate Crystals with Different Doping Ions, CMS Note 1997/054.

[9] X. Qu, L. Zhang and R.- Y. Zhu, Radiation Induced Color Centers and Light Monitoring for Lead Tungstate Crystals, CMS Note 1999/069

[10] G. J. Davies et al., Studies of Scintillation Light Collection in PbWO4 Crystals, CMS IN 1997/027

[11] The shower simulation to design the filter was done by A. Givernaud 